\title{
INGESTION DES MICROFILAIRES PAR LE VECTEUR DANS LE CAS D'UNE FILAIRE PÉRIODIQUE DITETALONEMA DESSETAE*
}

\author{
G. PETIT*
}

RÉSUMÉ. L'analyse, chez $D$. dessetae, de la périodicité de la microfilarémie, de l'ingestion des microfilaires par Aedes aegypti, et la comparaison avec l'ingestion de microsphères inertes inoculées au rongeur permettent de tirer les conclusions suivantes :

- Si les moustiques se gorgent pendant le plateau diurne, la densité des microfilaires ingérées est égale ou un peu supérieure à la densité des microfilaires dans le sang du sinus oculaire. Par contre, s'ils se gorgent la nuit, ils ingèrent 2,5 fois plus de microfilaires que ne le laisse prévoir la densité microfilarienne au sinus de l'œil ; il y a donc la nuit un phénomène de persistance des microfilaires dans la circulation sanguine cutanée du rongeur.

- Avec des moustiques de taille identique ingérant chacun un volume semblable de sang,la distribution des Aedes en fonction du nombre des microfilaires ingérées se rapproche d'une loi de Poisson $(\mathrm{K}=12)$, ce qui indique que la répartition des microfilaires dans le sang cutané du rongeur tend vers l'homogénéité. Cette répartition semble résulter d'un processus actif, car les microsphères inertes, à l'opposé des microfilaires, se distribuent d'une manière très hétérogène $(K=0,7)$ dans le sang du rongeur accessible à l'Aedes.

- L'ingestion par des moustiques de microfilaires ou de certains Plasmodium ne fait pas intervenir un phénomène de ralentissement de ces derniers dans les fins capillaires (moins de $10 \mu \mathrm{m}$ ) ; les moustiques semblent piquer dans des vaisseaux de plus gros calibre $(20-70 \mu \mathrm{m}$ de diamètre).

\section{Intake of microfilariae by the vector for a periodic filarioid, Dipetalonema dessetae.}

SUMMARY. The Filariid $D$. dessetae in its natural host, Proechimys oris, has a daily periodic microfilaraemia with a conspicuous peak during the day (blood from the ocular sinus).

- If the mosquitoes engorge during the daily peak, the density of microfilariae ingested is equal or slightly superior to the density of microfilaria in the blood from the ocular sinus. However, if they engorge during the night they ingest 2,5 times the number of microfilariae than the density of microfilariae in the ocular sinus indicates they should. Thus during the night there is a phenomenon of persistence of microfilariae in the cutaneous blood circulation of the rodent.

- With mosquitoes of similar size each ingesting a similar volume of blood the distribution of Aedes as a fonction of the number of microfilariae ingested resembles the Poisson distribution. This indicates that the distribution of microfilariae which are accessible to the vector in the blood of Proechimys is nearly homogeneous.

* Laboratoire de Zoologie (Vers), associé au CNRS, Muséum national d'Histoire naturelle, 61, rue Buffon, F75231 Paris Cedex 05.

I. Ce travail a pu être effectué grâce à une subvention de l'Organisation Mondiale de la Santé. Accepté le $\mathrm{I}_{5}$ janvier $\mathrm{I}_{98} \mathrm{I}$. 
- In comparison the inert microspheres are distributed heterogeneously in the cutaneous blood of the rodent $(\mathrm{K}=0,7)$. This apparently indicates that the microfilariae by their movements keep their homogeneous distribution.

- The small $(8-14 \mu \mathrm{m} \varnothing)$ and large $(18-24 \mu \mathrm{m} \varnothing)$ microspheres are ingested in the same way by Aedes or Anopheles. Thus the ingestion of "voluminous", objects in the blood such as microfilariae or certain Plasmodium does not require that the objects be slowed down in narrow capillaries. In fact it appears that the two vectors studied have access to vessels of a greater diameter (20$70 \mu \mathrm{m})$ than is generally believed.

\section{Introduction}

L'étude quantitative de l'ingestion des microfilaires a donné lieu à des travaux analysés récemment par Pichon et coll. (1975 et 1980).

Nous reprenons cette étude dans le cas de la Filaire Dipetalonema dessetae Bain, 1973, entretenue chez son hôte naturel Proechimys oris Thomas, 1904, et transmise expérimentalement par Aedes aegypti (souche Gkep). Cette Filaire présente un phénomène de périodicité journalière de la microfilarémie que nous décrivons; l'ingestion des microfilaires est étudiée sous trois aspects différents :

- L'ingestion en fonction des variations journalières de la microfilarémie.

- La distribution des Aedes en fonction du nombre de microfilaires ingérées.

- La comparaison de l'ingestion des microfilaires avec l'ingestion de microsphères inertes introduites artificiellement dans le sang de l'hôte.

\section{I - Ingestion des microfilaires et périodicité journalière de la microfilarémie.}

a) LA PÉRIODICITÉ

Pour étudier les variations de la microfilarémie, le sang est prélevé au sinus de l'œil du Proechimys, ce qui permet des ponctions régulières et suffisamment importantes. $50 \mathrm{~mm}^{3}$ de sang sont ainsi prélevés toutes les 3 heures pendant 24 heures chez 3 rongeurs différemment infestés (respectivement 3441, 1205 et 248 microfilaires pour $50 \mathrm{~mm}^{3}$ de sang à $12 \mathrm{~h}$ ).

La figure $7^{1}$ représente les variations de la densité microfilarienne pendant $24 \mathrm{~h}$; celles-ci sont identiques pour les 3 rongeurs étudiés. On distingue un plateau de forte microfilarémie entre $6 \mathrm{~h}$ et $15 \mathrm{~h}$ et un plateau de basse microfilarémie entre $18 \mathrm{~h}$ et $24 \mathrm{~h}$. La chute de la microfilarémie atteint pratiquement $90 \%$ de la microfilarémie maximum.

$20^{\circ} \mathrm{C}$ ). Expérience réalisée le $7 / \mathrm{ro} / 80$ à Paris dans les conditions du laboratoire (température $=$ 


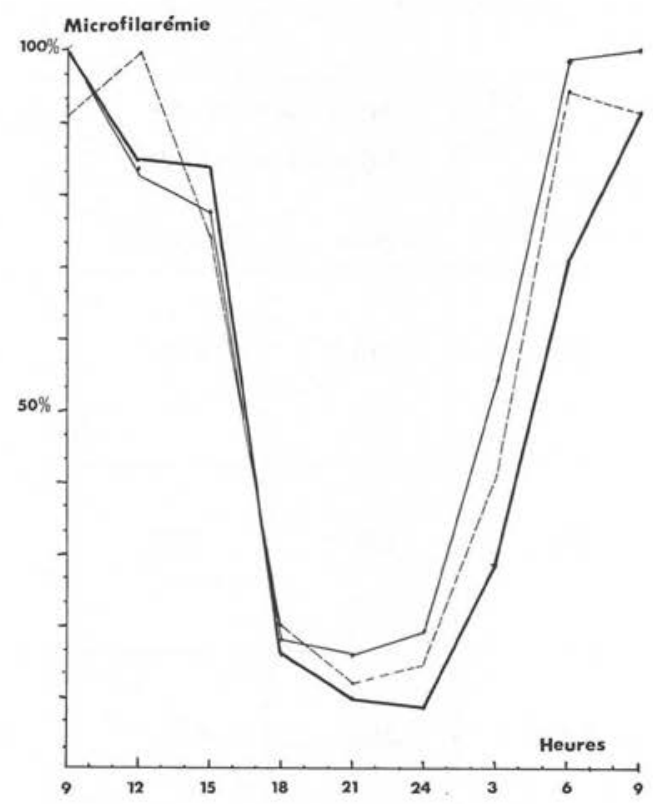

FIG. I. - D. dessetae : périodicité journalière de la microfilarémie chez 3 Proechimys (sang du sinus oculaire). Le nombre de microfilaires est exprimé en pourcentage du nombre maximum observé.

b) Ingestion des microfilaires pendant Le pLAteau diURne Et Le pLateau nocturne

Pour voir l'influence de ces fluctuations de la microfilarémie sur le nombre de microfilaires ingérées, des moustiques sont mis à gorger à $12 \mathrm{~h}$ et $22 \mathrm{~h}$ sur la peau ventrale de 2 Proechimys différemment infestés (respectivement 487 et 4105 microfilaires pour $50 \mathrm{~mm}^{3}$ de sang à $12 \mathrm{~h}$ ).

Les Aedes sont maintenus à $0^{\circ} \mathrm{C}$ après le gorgement pour éviter le passage des microfilaires dans l'hémocèle. L'estomac de chacun des moustiques est ensuite prélevé, dilacéré sur lame en goutte épaisse, qui sera colorée au Giemsa pour le comptage des microfilaires. Les résultats sont représentés tableau $I$.

Le nombre moyen attendu de microfilaires ingérées par Aedes est calculé par rapport à la densité microfilarienne au sinus de l'œil, chaque Aedes ingérant 4,5 $\mathrm{mm}^{3}$ de sang (Boorman, 1960).

- Si les moustiques se gorgent à $12 \mathrm{~h}$, la densité des microfilaires ingérées est égale ou un peu supérieure à la densité des microfilaires dans le sang du sinus oculaire (rapport 1,09 et 1,13).

- Par contre, s'ils se gorgent la nuit, ils ingèrent 2,5 fois plus de microfilaires que ne le laisse prévoir la densité microfilarienne au sinus de l'œil. 
TABLEAU I. - Ingestion des microfilaires de $D$. dessetae par $A$. aegypti pendant le plateau diurne et le plateau nocturne.

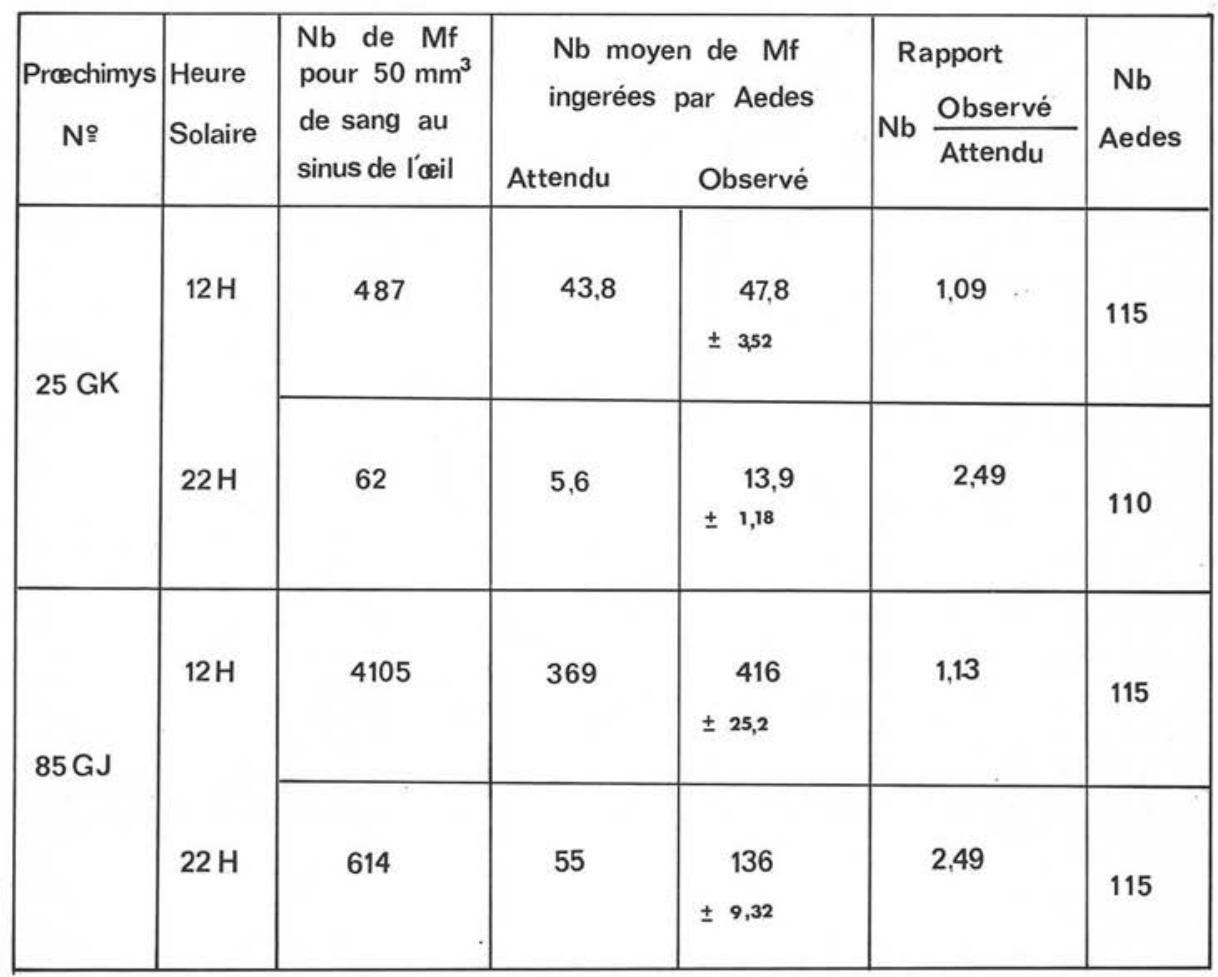

$(\mathrm{Nb}=$ nombre $; \mathrm{Mf}=$ microfilaire $; \mathrm{Nb}$ attendu $=$ nombre moyen théorique de microfilaires ingérées par Aedes, calculé en fonction de la microfilarémie au sinus de l’œil, chaque Aedes ingérant $4,5 \mathrm{~mm}^{3}$ de sang).

c) Discussion

La Filaire $D$. dessetae présente une périodicité journalière de la microfilarémie très marquée (presque $90 \%$ de chute journalière au sinus oculaire.) Elle est plus accentuée que chez Wuchereria bancrofti type pacifique et Dirofilaria immitis, où la chute ne dépasse pas $75 \%$ de la microfilarémie maximum; elle l'est moins que chez Wuchereria bancrofti et Loa loa, où la chute de la microfilarémie est pratiquement totale (Hawking, 1967).

Les relations différentes à $12 \mathrm{~h}$ et à $22 \mathrm{~h}$ entre le nombre attendu de microfilaires ingérées et le nombre observé montrent que la chute de la microfilarémie dans le sang cutané est beaucoup moins importante qu'elle ne l'est dans le sang du sinus oculaire. Lors de la chute de la microfilarémie, un certain nombre de microfilaires ne vont donc pas vers les poumons et persistent dans la circulation sanguine cutanée, soit qu'elles ne soient pas stimulées par les changements physiologiques de l'hôte, soit qu'elles se maintiennent activement malgré ce stimulus. Ce phénomène de rétention des micro- 
filaires dans les vaisseaux cutanés contredit un peu l'idée de l'adaptation d'une microfilaire à pic diurne à la piqûre par un insecte diurne ; cependant, la prise des microfilaires par l'Aedes reste nettement meilleure le jour (environ 3 fois meilleure pour les 2 expériences réalisées).

\section{II - Distribution des Aedes en fonction du nombre de microfilaires ingérées.}

a) Analyse des distributions

Les distributions obtenues antérieurement avec ce couple Filaire-vecteur (Petit, 1978) étaient surdispersées (ajustement à une loi binomiale négative, coefficient de surdispersion $\mathrm{K}$ égal à 3 ).

Actuellement, avec un élevage plus standard des moustiques (individus plus gros et de taille semblable) et un tri plus précis des femelles gorgées à réplétion, tous les Aedes font un repas de sang plus important et ingèrent chacun un volume semblable de sang. Dans ces conditions, on constate que les distributions se rapprochent d'une loi de Poisson (coefficient $\mathrm{K}$ atteignant la valeur de 12) (fig. 2).

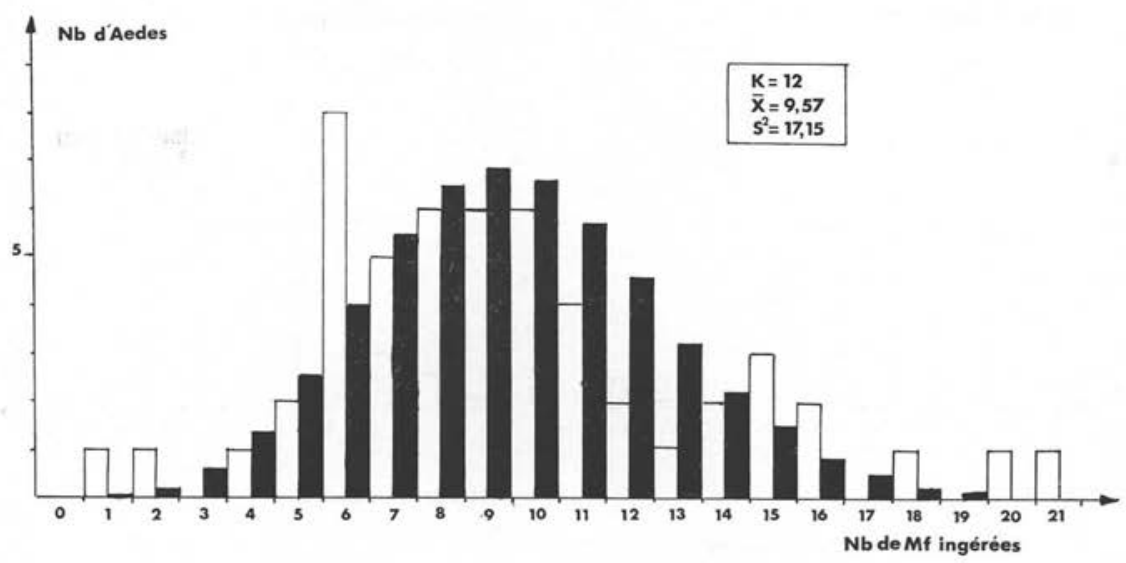

Fig. 2. - Distribution des Aedes en fonction du nombre de microfilaires ingérées. En blanc; distribution observée; en noir : distribution théorique de Poisson pour la même moyenne. (Coefficient de surdispersion $\mathrm{k}=\frac{\overline{\mathrm{x}}^{2}}{\mathrm{~s}^{2}-\overline{\mathrm{x}}} \overline{\mathrm{x}}$ étant la moyenne et $\mathrm{s}^{2}$ la variance).

b) Discussion

En parasitologie, les distributions des hôtes en fonction du nombre de leurs parasites sont toujours surdispersées (Crofton, 1971 ; May, 1977 ; Anderson, 1978), en particulier chez les vecteurs de Filaires (Schmid, 1972 ; Pichon et coll., 1975 et 1980). Cette surdispersion est particulièrement grande dans les distributions naturelles, où une faible partie des hôtes renferme la totalité de la population parasitaire. Très généralement le coefficient $\mathrm{K}$ est en effet bien inférieur à 3 . 
Par contre, dans les distributions expérimentales, il est possible, au moins pour notre couple Filaire-vecteur, d'obtenir une distribution plus homogène proche du modèle poissonnien en limitant les variations apportées par les fluctuations de la quantité de sang ingéré par les Aedes.

Ceci montre que la répartition des microfilaires dans le sang cutané du Proechimys tend vers l'homogénéité.

\section{III - Ingestion de microsphères inertes inoculées à un rongeur.}

a) Description des microsphères, modalités d'injection

Les particules sont constituées d'un polymère de Dextran, le Sephasorb HP (Pharmacia Fine Chemicals). Elles sont neutres chimiquement et ont un diamètre qui varie entre $8 \mu \mathrm{m}$ et $24 \mu \mathrm{m}$ pour un diamètre moyen de $14,7 \mu \mathrm{m}$ (fig. 3).

Ces microsphères sont injectées par voie intrapéritonéale $(3 \mathrm{~g}$ de Sephasorb dans $5 \mathrm{ml}$ de sérum physiologique pour un rat blanc de $100 \mathrm{~g}$ ). Elles entrent rapidement dans la circulation sanguine, leur densité passe par un maximum $24 \mathrm{~h}$ après l'injection,
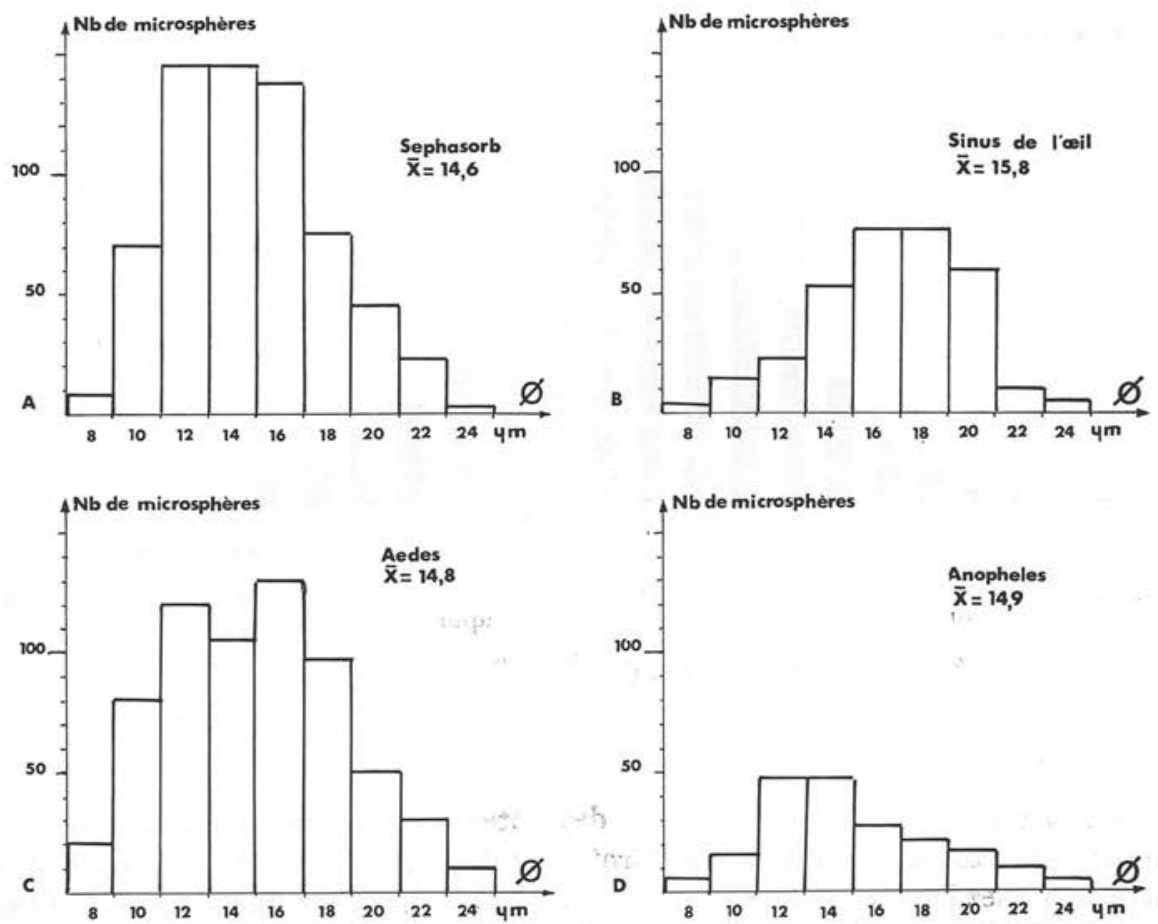

FIG. 3. - Histogramme de fréquence des microsphères en fonction de leur diamètre. A, dans le séphasorb pur; $\mathrm{B}$, dans le sang du sinus de l'œil ; C, dans le sang ingéré par les Aedes ; $\mathrm{D}$, dans le sang ingéré par les Anopheles. $(\mathrm{X}=$ diamètre moyen des microsphères, $\varnothing=$ diamètre des microsphères). 
puis elle décroît régulièrement. Une grande partie des microsphères ne pénètre pas dans la circulation sanguine et forme des kystes dans le péritoine ; d'autres semblent s'accumuler dans divers tissus et, en particulier, dans les tissus sous-cutanés.

Les microsphères sont repérables sur goutte épaisse simplement deshémoglobinisée, ou sur frottis non coloré.

b) TAILLE DES MicrosPHÈres INGÉrÉES

Une expérience d'ingestion de microsphères est réalisée sur un rat blanc $24 \mathrm{~h}$ après l'injection de Sepharsorb ; 104 Aedes sont gorgés sur la peau abdominale de l'animal comme lors des expériences d'ingestion de microfilaires ; de même, une prise de sang est effectuée au sinus oculaire du rongeur avant le gorgement.

Un test d'ingestion avec 30 Anopheles est réalisé parallèlement. L'histogramme de fréquence des différentes tailles de microsphères ingérées par l'Aedes ou l'Anopheles est représenté sur la figure 4.

Les Aedes et les Anophèles ingèrent dans les mêmes proportions les différentes tailles de microsphères trouvées dans le sang du sinus oculaire ou dans le Sephasorb pur.

c) Distribution Des «AEDES 》 EN FONCtION DU NOMBRE DE Microsphères INGÉRÉES

L'analyse est réalisée sur les 104 Aedes gorgés. Les caractéristiques de la distribution sont indiquées figure 4.

La distribution des Aedes en fonction du nombre de microsphères ingérées est très surdispersée, comme l'indique la valeur de son coefficient $\mathrm{K}$ égal à 0,7 , et s'ajuste bien à une loi binomiale négative.

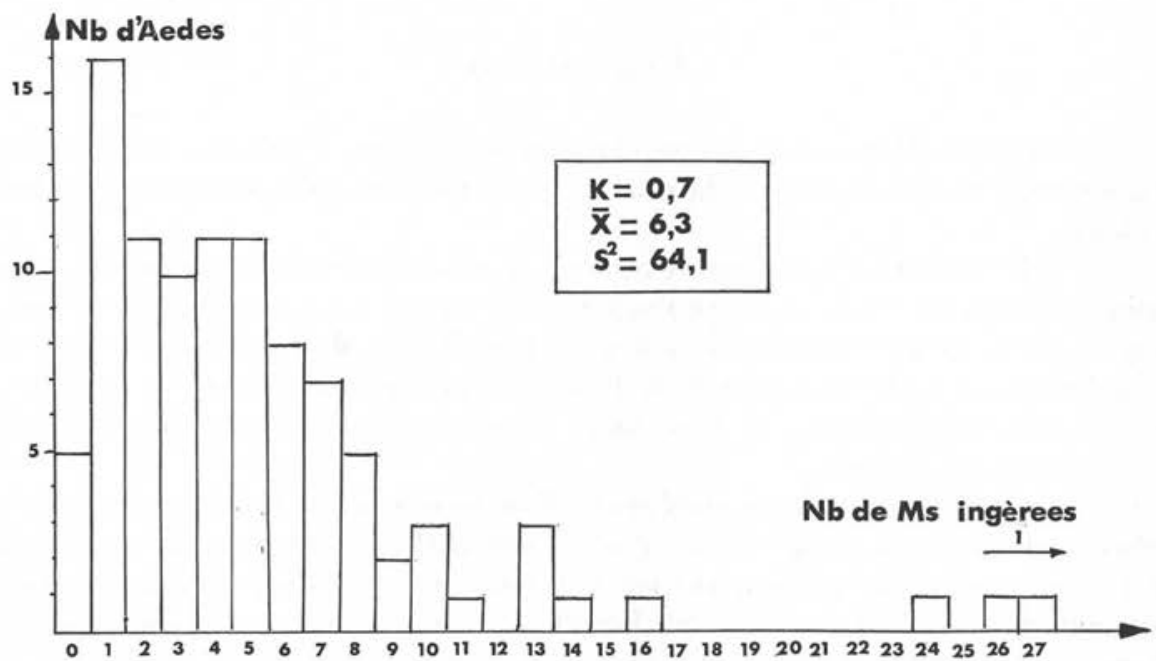

FIG. 4. - Distribution des Aedes en fonction du nombre de microsphères ingérées. (Ms $=$ microsphère, $\stackrel{1}{\rightarrow}=$ I Aedes ayant ingéré 49 microsphères). 
d) Discussion

L'examen de l'histogramme ( $f i g$. 4) montre que l'Aedes ou l'Anophèle n'ingère pas préférentiellement les particules les plus grosses. Cette idée était suggérée par les travaux de plusieurs auteurs : Pichon et coll. (1975 et 1980) et nous-mêmes (1979) évoquons le ralentissement des microfilaires lors de leur passage dans les fins capillaires (diamètre inférieur à $10 \mu \mathrm{m}$ ) pour rendre compte des surdispersions observées lors de l'ingestion; Landau (1980) fait intervenir la grande taille de certains gamétocytes (diamètre compris entre 9,5 et $10,5 \mu \mathrm{m}$ ), plus ralentis dans les fins capillaires pour expliquer leur concentration chez les Anophèles.

Ces hypothèses paraissent donc erronnées; il semble que l'Aedes ou l'Anopheles aient accès à des vaisseaux de plus grosse taille qu'on ne l'imagine généralement ; la longueur de leur trompe et son enfoncement lors de la piqûre font penser qu'ils prélèvent du sang entre 400 et $1500 \mu \mathrm{m}$ de profondeur, c'est-à-dire au niveau de vaisseaux dont le diamètre varie entre $20 \mu \mathrm{m}$ et $70 \mu \mathrm{m}$ (artérioles, veinules, anastomoses artério-veineuses) et non pas dans les très fins capillaires superficiels. D'autre part, le volume du repas de sang d'un Aedes ( $4,5 \mathrm{~mm}^{3}$ environ) indique que, même s'il pique dans un fin capillaire, il prélève principalement du sang plus profond; en effet, cette quantité de sang ingéré correspondrait au volume de sang compris dans un capillaire de $10 \mu \mathrm{m}$ de diamètre sur $12 \mathrm{~m}$ de long, alors que leur longueur ne dépasse pas $800 \mu \mathrm{m}$ chez les mammifères (Chèvremont, 1956).

L'analyse de la distribution des Aedes en fonction du nombre de microsphères ingérées montre une forte surdispersion ( $\mathrm{K}=0,7)$, ce qui indique qu'une particule inerte tend, à l'opposé des microfilaires, à se répartir d'une manière hétérogène dans le sang accessible au vecteur.

\section{Conclusions}

- La Filaire $D$. dessetae présente chez son hôte naturel $P$. oris une microfilarémie périodique journalière avec un plateau diurne de forte microfilarémie (sang du sinus oculaire).

- Si les moustiques se gorgent pendant le plateau diurne, la densité des microfilaires ingérées est égale ou un peu supérieure à la densité des microfilaires dans le sang du sinus oculaire. Par contre, s'ils se gorgent la nuit, ils ingèrent 2,5 fois plus de microfilaires que ne le laisse prévoir la densité microfilarienne au sinus de l'œil ; il y a donc la nuit un phénomène de persistance des microfilaires dans la circulation sanguine cutanée du rongeur.

- Avec des moustiques homogènes (individus de même taille ingérant chacun un volume semblable de sang), la distribution des Aedes en fonction du nombre des microfilaires ingérées s'ajuste à une loi de Poisson, ce qui indique que la répartition des microfilaires dans le sang du Proechimys accessible au vecteur tend vers l'homogénéité.

— A l'opposé, des microsphères inertes se répartissent d'une manière très hété- 
rogène $(K=0,7)$ dans le sang cutané du rongeur, ce qui semble indiquer que les microfilaires par leurs mouvements s'y maintiennent activement d'une manière homogène.

- Les petites microsphères (8-14 $\mu \mathrm{m}$ de $\varnothing)$ et les grosses microsphères (18-24 $\mu \mathrm{m}$ de $\varnothing)$ sont ingérées de la même manière par les Aedes ou les Anophèles; l'ingestion de parasites sanguins " volumineux ", tels que des microfilaires ou certains Plasmodium, ne fait donc pas intervenir un phénomène de ralentissement de ces derniers dans les fins capillaires. Il semble, en fait, que les 2 vecteurs étudiés aient accès à des vaisseaux de plus gros calibre $(20-70 \mu \mathrm{m})$ qu'on ne l'imagine généralement.

\section{BIBLIOGRAPHIE}

ANDERson R. M : The regulation of host population growth by parasitic species. Parasitology, 1978,76, II9-1 57 .

Boorman J. P. T. : Observations on the feeding habits of the mosquito Aedes (Stegomyia) aegypti (Linnaeus); The loss of fluid after a blood meal and the amount of blood taken during feeding. Ann. Trop. Med. Parasitol., 1960, 54, 8-14.

ChÈvremont M. : Notions de cytologie et histologie, Éd. Desoer, I960, 991 p.

CROFTON H. D. : A quantitative approach to parasitism. Parasitology, 1971, 62, 179-193.

HAWKING F.: The 24-hour periodocity of microfilariae : biological mechanism responsible for its production and control. Proc. $R$. Soc, B, $1967,169,59-76$.

landau I., Miltgen F., Boulard Y., Chabaud A. G., Baccam D. : Études sur les gamétocytes des Plasmodium du groupe «vivax» : morphologie, évolution, prise par les Anophèles et infectivité des microgamétocytes de Plasmodium yoelii. Ann. Parasitol. Hum. Comp., 1979, s4, I45-I6r.

MAY R. M. : Dynamical aspects of host-parasite associations : Crofton's model revisited. Parasitology, 1977, 75, 259-276.

PetrT G. : La Filaire Dipetalonema dessetae : phénomènes de régulation et rendement parasitaire chez 1'Aedes vecteur. Ann. Parasitol. Hum. Comp., 1978, 53,649-668.

Pichon G., ProD'Hon J., RIVIÈre F. : Distribution des microfilaires ingérées par les moustiques. WHO. FIL $/ 75.139,1975,24$ p.

PIchoN G., PROD'HON J., RIVIĚRE F. : Hétérogénéité de l'ingestion des parasites sanguicoles par leurs vecteurs : description quantitative et interprétation. C. R. Acad. Sci. Paris, 1980, 290 , sér. D, IOII-IOI3.

Schmid W. D., Robinson E. J. : The pattern of a host-parasite distribution. J. Parasitol., 1972, 58, 907-910. 